\title{
Volunteer Studies Replacing Animal Experiments in Brain Research
}

\author{
Report and Recommendations of a Volunteers in Research \\ and Testing Workshop
}

\author{
Gill Langley, 1 Graham Harding, ${ }^{2}$ Penny Hawkins, ${ }^{3}$ Anthony Jones, ${ }^{4}$ Carol \\ Newman, ${ }^{1}$ Stephen Swithenby, ${ }^{5}$ David Thompson, ${ }^{6}$ Paul Tofts ${ }^{7}$ and \\ Vincent Walsh8
}

\begin{abstract}
1Dr Hadwen Trust for Humane Research, 84a Tilehouse Street, Hitchin, Hertfordshire SG5 2DY, UK; ${ }^{2}$ Psychology Institute, Life and Health Sciences, Aston University, Aston Triangle, Birmingham, West Midlands B4 7ET, UK; ${ }^{3}$ Royal Society for the Prevention of Cruelty to Animals, The Causeway, Horsham, West Sussex RH12 1HG, UK; ${ }^{4}$ Rheumatic Diseases Centre, Clinical Sciences Building, Hope Hospital, University of Manchester, Eccles Old Road, Salford, M6 8HD, UK; ${ }^{5}$ The Open University, Walton Hall, Milton Keynes, Buckinghamshire MK7 6AA, UK; ${ }^{6}$ Section of Gastrointestinal Science, Clinical Sciences Building, Hope Hospital, University of Manchester, Eccles Old Road, Salford M6 8HD, UK; ${ }^{7}$ NMR Research Unit, Institute of Neurology, University College London, Queen Square, London WC1N 3BG, UK; ${ }^{8}$ Department of Experimental Psychology, University of Oxford, South Parks Road, Oxford OX1 3UD, UK
\end{abstract}

\section{Introduction}

National (1) and European (2) legislation requires the replacement, reduction and refinement of animal experiments wherever feasible, placing a responsibility on scientists and others to implement these concepts in the planning, design, approval and conduct of research.

There has been little discussion of the role of human volunteer studies in replacing and reducing animal experiments. Following its conference in Birmingham, UK, in 1995 (3), the initiative called "Volunteers in Research and Testing" continues to explore the potential of ethical volunteer studies as alternatives to animal experiments. As part of this initiative, a workshop was held in November 1998 to study the field of brain research.

During the $1990 \mathrm{~s}$, the development of imaging techniques opened new windows into the human brain, revolutionising research by permitting studies which had not previously been possible. Imaging methods offer insights into brain structure and function at the cell-population and systems levels, and one of their strengths is that they permit a more global, integrated view of the human brain.

However, replacing animal experiments requires more than technology: it also needs a commitment by the scientific community and by funding agencies. Such a commitment is bound to include a reconsideration of the ways in which questions are asked about brain structure and function, and the kinds of answers which are considered acceptable. Imaging and similar techniques offer a new and, perhaps, slightly different route to understanding the brain.

The remit of the workshop was:

- to consider what questions are asked in brain research conducted on animals and what kinds of answers are obtained;

- to assess whether human studies can already provide equivalent data in some areas;

- to review the questions addressed by human studies and the kinds of answers obtained; 
- to review the technical and other limitations in studying humans and how they might be overcome; and

- to recommend how the replacement and reduction of animal-based brain research with ethical human studies can be advanced.

Participants were asked to work on the assumption that studies of patients and healthy volunteers are conducted ethically according to international guidelines and best practice. We acknowledge that there are important unresolved controversial issues con- cerning, for example, the validity of informed consent, but these were not within the remit of this workshop. Because the specific aim was to identify how human studies could partly or wholly replace animal experiments, the worishop excluded from discussion areas in which animals are not currently used, such as language research. Imaging studies involving animals, which may be a means of refining but not replacing animal experiments, were also outside the workshop's remit.

The participants included scientists committed to the replacement of animal experiments and researchers involved in human

Table I: Characteristics of techniques used in human brain research

\begin{tabular}{llllll}
\hline Technique & $\begin{array}{l}\text { Spatial } \\
\text { resolution }\end{array}$ & $\begin{array}{l}\text { Temporal } \\
\text { resolution }\end{array}$ & Measures & Invasiveness & Cost \\
\hline PET & $\begin{array}{l}3-5 \mathrm{~mm} \\
\text { any depth }\end{array}$ & $10-60$ seconds & $\begin{array}{l}\text { Radiolabelled } \\
\text { ligands }\end{array}$ & $\begin{array}{l}\text { Short half-life } \\
\text { radiation, } \\
\text { scans limited }\end{array}$ & Very expensive \\
\hline SPECT & $\begin{array}{l}\text { 6mm } \\
\text { any depth }\end{array}$ & -10 minutes & $\begin{array}{l}\text { Radiolabelled } \\
\text { ligands }\end{array}$ & $\begin{array}{l}\text { Short half-life } \\
\text { radiation, } \\
\text { scans limited }\end{array}$ & Less expensive \\
than PET
\end{tabular}

$E E G=$ electroencephalography; $M E G=$ magnetoencephalography; $M R I=$ magnetic resonance imaging; $F M R I=$ functional magnetic resonance imaging; $M R S=$ magnetic resonance spectroscopy; PET = positron emission tomography; SPECT = single photon emission computed tomography; TMS = transcranial magnetic stimulation 
volunteer studies in several fields, employing a range of imaging and related techniques (see Table I), including positron emission tomography (PET), single photon emission computed tomography (SPECT), magnetic resonance imaging (MRI), functional magnetic resonance imaging (fMRI), magnetic resonance spectroscopy (MRS), magnetoencephalography (MEG), electroencephalography (EEG) and other event-related potentials, and transcranial magnetic stimulation (TMS).

\section{Brain Research on Animals}

In 1998 (4), 45,992 animal procedures (twothirds of them involving rats) conducted in Britain involved interference with the brain and 13,224 procedures (mainly with rats and mice) involved interference with the organs of the special senses (for example, vision, taste and smell). Although rats and mice comprised most of the animals used, other species were also used, including other rodents, rabbits, cats, dogs, ferrets, ungulates, monkeys, birds, reptiles, amphibians and fish.

Monkeys are frequently chosen for research into cognitive and sensorimotor functions, and are used as "models" of human neurological and psychological conditions. In Britain, in 1998, $16 \%$ of all experiments on monkeys (i.e. 594 procedures) involved studies of the central or peripheral nervous system or of the special senses. It is especially difficult to provide for the social, physical, mental and emotional needs of primates in laboratories. For these reasons, replacing brain research on primates was a major, although not the sole, focus of the workshop discussions.

\section{What Questions are Asked in Brain Research on Animals and What Kinds of Answers are Obtained?}

The fields of animal research considered at the workshop included: studies of cognitive functions (such as memory, learning, attention); sensory functions (for example, pain, vision, hearing and taste) and motor activities (for example, micturition, swallowing and arm movement) and sensorimotor integration; brain plasticity and reorganisation (for example, after trauma); brain pathologies (such as stroke, multiple sclerosis, Parkinson's disease and epilepsy); and central nervous system (CNS) drug development (including drug disposition, the blood-brain barrier, drug metabolism, receptor occupancy, efficacy and toxicity), or post-marketing drug investigations.

What questions are asked in animal research?

Animal-based brain research is wide ranging, because it is considered to involve fewer ethical constraints than do human volunteer studies. Many techniques are used to address questions of brain structure and function in animals and, naturally, these have tended to direct the types of questions asked and the kinds of answers obtained.

Electrophysiology with intracellular or field electrodes is common to animal studies of cognition, sensory and motor functions, neural connectivity and brain disorders. Electrode recording and stimulation are used to locate and explore the function of active areas of the brain or individual neurons. Examples include a study of the effects of novel or familiar stimuli on neuronal activity in the amygdala of monkeys performing recognition memory tasks (5), or associating single brain neurons with specific activities such as face recognition or taste specificity.

Lesioning techniques involving electrodes, aspiration or neurotoxins are used to localise functions in particular brain centres. Lesioning is frequently used in research into cognitive, sensory and motor functions. An example might involve training primates to perform a motor task. A small region of the motor cortex is destroyed by aspiration or injection of toxin, and the animals are then retested. Lesioning can identify which brain regions are critical to certain functions, but does not provide information about the relative timing of activity in various brain areas, or about regions which are not essential to a task but are nevertheless engaged during its performance. Specific kinds of lesions are also employed in animal models of certain human disorders, such as stroke and Parkinson's disease.

Fluorescent or other tracers are used in vivo in animals to identify anatomical connections within the brain, at the level of single fibres or fibre tracts. Tracers are injected into the living animal, followed by post-mortem analysis to demonstrate connections between regions of the brain, such as the auditory cortex and the frontal lobe in macaques (6). Another example is tracing retinal projections through the optic system in the brains of Japanese monkeys by 
injection of a cholera toxin subunit into the eye (7).

Biochemical and pharmacological methods are applied to cognitive, sensory and motor studies in animals, as well as in researching brain dysfunction and drug development. For example, with brain-lesioned rhesus monkeys in Parkinson's disease research, microdialysis probes have been used to sample neurotransmitter and metabolite levels in small regions of the brain (8). Micro-injection of drugs or neurotransmitters is another technique used, for example, in a study to identify the role of D1 dopamine receptors in spatial working memory function in the rat (9).

In animal experiments, the regional, cellular and subcellular distribution of receptors can be studied by injecting receptor-specific antibodies and analysing antibody labelling in post-mortem brain tissue (10). Receptors involved in pain perception or drug dependence, for example, can be identified and mapped. Various levels of neurotransmitter uptake or metabolism, or the receptor binding of a putative central nervous system drug or other ligand, can be measured.

Imaging and related techniques such as MRS, MRI, PET and SPECT are also used in animal studies, not only in neurochemistry and neuropharmacology, but in most of the approaches described above.

\section{What hinds of answers do animal studies} provide?

In animal research, questions about "where" and "how" the brain performs its activities, or fails to do so, can be asked and answered at the subcellular, single-cell and systems levels.

With the advent of non-invasive imaging, which enables the human brain to be studied at a resolution of a few millimetres, the strength of animal research may be perceived as being at the single-cell level. Of course, it is not necessary to measure the response of every individual neuron in order to understand functional areas of the human brain and, indeed, knowledge at the level of an individual neuron does not always assist in interpreting brain activity at a level of many thousands of neurons, which is the scale of the neural networks involved in most tasks. Moreover, in the case of medical research rather than fundamental research, the precision of animal studies may be superfluous if the results are not reliably transferable to humans because of species differences.
Animal experiments include some invasive studies of brain pathology and some experimental interventions aimed at treating dysfunctions, which are not feasible in volunteers. For example, in animal models of stroke, the region of brain ischaemia can be correlated experimentally with particular behavioural impairments. However, animal models seldom express the complexity of human disorders. A case in point is multiple sclerosis, where animal experiments sug. gested a relatively simple picture of demyeli. nation causing disability. Imaging studies of patients with multiple sclerosis have now revealed a greater complexity: remyelination may occur and axonal death may play an important role (11). Finally, tests for drug toxicity are commonly performed in animals because of obvious ethical constraints on the use of volunteers.

Can Human Studies with Imaging and Related Methods Already Provide Equivalent Data in Some Areas of Brain Research and, if so, Which?

\section{Cognitive functions}

In the field of cognitive studies, human volunteer research has enormous potential to replace animal experiments. This is illustrated by research into memory. In monkeys, a prefrontal cortical region centring on the principal sulcus is considered to be important in working memory for spatial locations. Recording and stimulation experiments with microelectrodes identified the location and function of the specialised area in monkeys, but there was dispute about the site and even the existence of such a region in humans. Susan Courtney and her colleagues used fMRI to investigate the location of the spatial working memory area in humans (12). An area was identified that is specialised for this task, but in a more superior and posterior location than in monkeys, which may explain why it was not discovered in earlier human studies.

In cognitive psychology, most brain surface lesioning studies and many regional activation experiments with electrode mats in animals could be replaced by human studies with TMS, and this work is already under way. TMS can be used to create very short-lived and reversible "lesions" in the brains of volunteers (13). An advantage of 
TMS is that the experimental lesion is so brief that the brain does not have an opportunity to reorganise, which can be a limitation in conventional animal experiments. Other advantages are reversibility, the ability to study timing, and the ability to use within-subject controls. Although TMS does not penetrate deeper structures and does not have a high spatial resolution, the latter can be improved by combining TMS with other imaging techniques (Figure 1).

PET scans with radiolabelled ligands have enabled studies to be made of neurotransmitter fluxes in volunteers during behavioural tasks. Volunteers played a video game, which involved learning to navigate a tank through a battlefield, while undergoing PET scans (14). The task was similar to those performed in animal studies in which dopamine is released during the anticipatory stage of motivated behaviour. Radiolabelled raclopride was used to bind to D2 receptors in the brains of the volunteers. During the performance of the task, raclopride binding was reduced significantly, indicating an increased release and binding of dopamine to its receptors. This was the first study to demonstrate the behavioural conditions in humans under which dopamine is released, and illustrated the ability of PET to detect neurotransmitter fluxes in the active human brain.

Whereas imaging techniques cannot achieve the spatial resolution of microelectrode recordings from single brain cells, in humans the presence of these cells can be inferred by other means, including analysing results from MEG, MRI and PET studies. Data from imaging of patients with dysfunctions or accidental brain damage are also useful. In future, the question which has to be answered is whether it would be ethically justifiable to verify human data by doing electrode studies in animals; the workshop participants took the view that it would not be justifiable.

One limitation to cognitive studies in humans has been the lack of non-invasive techniques for tracking neuronal connections between brain regions. Detailed connectivity studies have only been possible by using invasive tracer methods in animals or, more indirectly, in post-mortem human brain by studying demyelination patterns to determine clinical-pathological correlations. A recent breakthrough will open new avenues in tract-tracing in human volunteers: US scientists have developed an MRI method that tracks the direction of fastest diffusion of water, thus delineating fibre trajectories (15). They successfully followed fibre bundles over long distances and in a number of regions of the living human brain, obtaining results which were consistent with existing data. The method also identified previously undescribed topologies in other pathways, and will allow the study of fibre connections among functionally and anatomically defined brain areas in individual subjects.

The workshop participants concluded that human studies are feasible and appropriate for studying both the systems level and the global level in psychology. The main limit to human studies in this field, compared with animal experiments, is spatial resolution; the very localised electrode studies in animals cannot be conducted in humans. If animal experiments in psychology were to stop, there would be a loss of some fine-grained neurodynamics; however, psychology is the "macro" science of the brain and species differences can be critical. Most of the participants felt that human studies could provide virtually all the data necessary in this field.

\section{Sensory and motor functions}

Human volunteer research into these areas makes use of several techniques, and using imaging modalities with complementary temporal and spatial resolutions can yield good results. In particular, the constellation of regions working together on different aspects of a function can be studied in the human brain by imaging methods.

As an illustration, in visual processing studies, fMRI scans provide an anatomical substrate for functional localisation by other imaging methods such as MEG and EEG. With MEG, the chronological sequence of activation of different brain areas can be followed in real time over milliseconds, replicating the temporal sensitivity of animal experiments. Such studies have provided evidence of the localisation, temporal dynamics and functional response of area V5 in humans (16; Figure 2).

Other studies have investigated the sensorimotor modulation of human cortical swallowing pathways, by using TMS over the motor cortex to activate cortical projections to the pharynx and oesophagus (17). It was 
found that the swallowing motor pathways from each cortical hemisphere interact, and that their excitability is modulated both in the brain stem and in the cortex by sensory input.

Another productive way of combining imaging methods allows the visualisation of functional networks in the human brain. TMS is used to stimulate an area of the brain with a known function; connections, some of which may have been undiscovered by electrophysiology in animals, are activated and can then be visualised as increases in cerebral blood flow by using PET. An example is a simultaneous study of volunteers with TMS stimulation and PET scanning, which showed correlations between the left frontal eye field and the visual cortex of the superior and medial parieto-occipital regions (18).

In view of in vitro data suggesting that blocking the uptake of $\gamma$-aminobutyric acid (GABA) modulated inhibition of the motor cortex, animal experiments would have been one option for pursuing this hypothesis in vivo. However, a TMS study was conducted in volunteers who took a drug which blocks GABA uptake (19). This provided evidence that GABA uptake blockage differentially modulates motor cortex inhibition: presynaptic GABA autoreceptors may be involved.

fMRI machines with high magnetic field strengths have good spatial resolution, and the most powerful equipment is capable of resolving ocular dominance columns approximately $1 \mathrm{~mm}$-diameter columns of neurons in the visual cortex. This level of resolution will permit investigations of the many columns of neurons elsewhere in the brain. Microelectrode studies are occasionally possible in patients about to undergo brain surgery, such as patients with epilepsy who are being monitored with implanted electrodes (20). There are ethical restrictions on this kind of work, as surgery may be prolonged as a result.

The analysis of human post-mortem brain tissue can also contribute to understanding structure and function. Cytochrome oxidase histochemistry has been used to study the modular organisation of the human extrastriate visual cortex, by comparing the patterns of cytochrome oxidase (a measure of local metabolic activity) in normal postmortem brain and in brain from patients with macular degeneration (21). Moreover, a report published in 1998 demonstrated, for the first time, that axonal connections can be traced in post-mortem human brain, up to eight hours after death (22). Neurons provided with suitable in vitro conditions retained active transport functions, and tracers were followed over distances of $0.5-1.5 \mathrm{~cm}$ from the point of application. This work opens up new possibilities for the study and culture of human post-mortem neurons, which would complement the in vivo imaging approaches.

\section{Plasticity and repair}

In volunteers affected by brain injury, limb amputation or stroke, simultaneous PET scanning and TMS permit the study of how the brain reorganises and how rehabilitation develops. For example, some stroke patients regain the use of a limb despite irreversible damage to the original part of the cortex responsible for motor control of that limb (23). Combining TMS with PET or fMRI could identify the new pathways used by the brain.

TMS has also been used in combination with sensory stimulation to study cortical plasticity in volunteers. Healthy volunteers swallowed an electrode inside a catheter, and changes in cortical plasticity were then monitored with TMS. Sensory stimulation of the pharynx lowered the excitability threshold of the area of motor cortex involved in swallowing and increased the area of the cortex representing the pharynx (24). This indicates cross-modal plasticity between the sensory and motor systems, and also suggests that pharyngeal stimulation may assist recovery in stroke patients suffering from an inability to swallow. It is easy to conceive of invasive animal experiments being conducted to achieve a similar result.

Sometimes plasticity changes can be measured in post-mortem human brains or in biopsy samples. Ultrastructural studies of such samples from patients with Alzheimer's disease $(A D)$ and from age-matched controls showed that, in specific areas, the number of synapses had decreased in AD patients (25). As synaptic density declined, synapse size increased, suggesting a degree of plasticity which may partly ameliorate the fall in synapse numbers seen in $A D$.

These kinds of human data compare favourably with those from animal experiments, where projections from one part of the brain to another have conventionally 
been determined by using tracers or dyes followed by post-mortem analysis. The advantage of the human imaging studies described is that the living brain can be studied in action, and the results are of ultimate relevance.

\section{Brain pathology}

When studying brain disorders, the scope for research in healthy volunteers is limited. However, long-term serial imaging of patients may be required for diagnosis or monitoring purposes, and the results are proving fruitful in several research fields.

Magnetic resonance (MR) techniques can be used with patients to study the underlying pathophysiology and biochemistry of numerous disorders including stroke, epilepsy, multiple sclerosis (MS), HIV-associated neurological effects, brain tumours, brain trauma and metabolic diseases. Research into stroke and brain ischaemia has involved rats, gerbils and monkeys, but the lesions produced may be different from those in humans. It is increasingly possible to study stroke patients by using MR-measured parameters such as brain capillary permeability, interstitial volume and mean transit time of blood flow.

For many years, experimental allergic encephalomyelitis was the standard animal model of MS, but it is not very representative of the pathology of the human illness. MR techniques, such as dynamic MR with contrast enhancers and magnetisation transfer imaging, provide information on the permeability of the blood-brain barrier, which is disrupted in MS, and in the presence of myelin. Studies of disease progression in patients have already provided a better understanding of the nature of MS lesions.

Other conditions whose natural history can now be researched in patients, rather than in animal models, include brain tumours, dementia and depression. Using SPECT with a ligand which binds to dopamine transporter sites, an experiment tested the hypothesis that patients with depression have lower densities of dopamine transporter in the basal ganglia (26). Unexpectedly, the reverse was found to be true. This cast doubt on the prevailing assumption that reduction in dopamine transmission in depression leads to secondary down-regulation of dopamine transporter density.
SPECT, spatially co-registered with MRI, was used to measure regional cerebral blood flow in epileptic patients suffering from ictal vomiting (27). The study suggested that acti vation of a complex cortical network, involving the medial and lateral superior aspects of the temporal lobe, is responsible for ictal vomiting

PET studies of Parkinson's disease in volunteers (28) have used various radiolabelled ligands to visualise presynaptic dopamine insufficiency, to measure dopamine reuptake sites in early and later stages of disease, and to demonstrate that the drug selegiline inhibits monoamine oxidase- $B$ in the cortex.

MRS offers a non-invasive means of measuring the distribution and concentration of several neuronal and glial cell markers and relating these to human brain function in health and disease. Glial cell integrity and myelination, neuronal development and loss, disturbed energy production, and cell membrane turnover, are among the features that can be measured by MRS.

\section{Drug development and related investigations}

Imaging of the human brain can already be of immense value in developing CNS drugs, although its potential has yet to be fully explored by pharmaceutical companies. Receptor sub-types, enzymes and neurotransmitter carriers may all be targets of drugs for treating psychiatric and neurological disorders.

Imaging studies with volunteers can reduce the scale and duration (and therefore the costs) of CNS drug trials, as well as reducing animal experiments. Human PET studies with sub-toxic and sub-therapeutic doses (in the picomole range) of a radiolabelled drug need minimal prior animal toxicity data and have a low risk of side-effects $(29,30)$. To illustrate this, quite early in the development of a centrally-acting drug, PET studies with volunteers can identify whether and how much of the drug reaches its target and which receptors are affected. By pinpointing the most promising drug candidates, such studies will reduce the number of animal tests. Even tracer doses of an experimental drug can yield qualitative and quantitative information on drug metabolites. Optimal doses for clinical trials can be predicted on the basis of PET measurements of receptor occupancy, achieving a balance 
between beneficial effects and side-effects This should facilitate clinical trials, as well as reducing preclinical studies in animals

For example, the efficacy testing of centrally acting analgesics could be made more rational and more cost-effective by introducing early studies in humans. Currently, the development of a new analgesic involves efficacy testing in animal models before phase I clinical trials. At this stage, dose selection is partly guesswork and if the candidate drug does not work in humans, a substantial company investment is lost. In the development of enkephalinase inhibitors, one candidate drug was very effective in rodents (31) but did not appear to be active as an analgesic in humans, probably because of poor uptake into the brain. This was not discovered until clinical trials, after approximately $£ 20$ million had been invested by the company

A different approach would have been to determine drug disposition early on, in the species of interest. The use of a carbon-11labelled molecule and PET imaging with vol unteers would have determined whether the drug penetrated the blood-brain barrier Then, further studies could have followed, to ascertain whether the analgesic affected the target areas of the human brain.

Sometimes, safety concerns about CNS drugs arise after they have been on the market for some time. One approach, which might yield answers to those concerns, is to perform additional tests in animals, but the results may be species-specific. A newer and more relevant approach is to conduct postmarketing tests in volunteers. A case in point was a study to measure levels of the appetite-suppressing drug, dexfenfluramine, in the brains of patients being treated clinically for obesity (32). Animal tests had indicated that high levels of the drug caused irreversible neurochemical changes, but using MRS with a radiolabelled ligand showed that these levels were not reached in the human brain.

\section{What Questions are Addressed by Human Studies and What Kinds of Answers are Obtained?}

In medical research, animal experiments may provide data of considerable precision but of variable accuracy, in terms of relevance to the human brain. Human imaging research may produce qualitatively different data from those of animal experiments, which some scientists find difficult to accept. However, human research overcomes the species barrier - especially significant in drug responses and in functional anatomy and human data are of ultimate relevance in medical or clinical research. In these respects, if the answers provided by human studies are different from those of animal studies, this may be beneficial.

Human subjects are easier to train to perform various tasks: it can take months to train monkeys, so human studies can be faster. Some studies, such as those of cognitive and sensory functions, including vision and pain sensation, also benefit from the advantage of communicating with the research subjects. In pain research, human volunteers can be asked whether they are in pain, but pain can only be inferred in experiments with animals.

Inevitably, the techniques used affect the type of data acquired. Imaging the human brain has provided new global information about integrated networks, and has identified areas of unexpected relevance, which have taken some research fields beyond the scope of animal studies. For example, before the advent of imaging, vision research relied on studies of accidental brain damage in humans and on animal experiments. Functional imaging has filled in missing anatomical and functional detail, and knowledge of the visual cortex has increased dramatically with human research

In pain research, until imaging techniques were applied to humans, it was unclear whether the cortex is even involved in pain perception, and the roles of various cortical areas in pain perception were not identified. PET and other functional imaging methods have identified the key human areas involved in pain processing, and interactions between them, for example, in rheumatic pain (33). Whereas animal work has been focused on specific cortical areas, which limited knowledge, human studies have broadened it and have opened up new opportunities for better understanding.

Human studies employing TMS to create a reversible lesion provide better temporal resolution than brain lesioning in animals, which does not supply information on the relative timing of activities or on how brain areas for, say, perceiving colour, form and 
movement, interact. Additionally, the reversibility of TMS effects permits studies to be repeated, and human subjects can be used as their own controls, permitting within-subject comparisons not available with the traditional lesioning method.

Imaging of patients can offer scientific advantages over the study of animal disease models. Being relatively non-invasive, longterm serial imaging can be conducted where it is required for a patient's benefit. In animal research, where disease conditions are inflicted on otherwise healthy animals, longterm or recovery experiments may not be permitted, because of ethical constraints.

In drug development, early imaging studies with volunteers can be used to assess the efficacy and metabolism of CNS drugs, without problems of species variation, which would minimise the scale and duration of drug trials as well as reducing animal tests.

\section{What are the Present Limitations to Human Studies and What are the Prospects of Overcoming Them?}

\section{Scientific and technical limitations}

There are scientific and technical limitations to any method, and imaging is no exception. Some of these are inherent in the process; for example, there will always be temporal limits with PET and SPECT. There is little prospect of improving the spatial resolution of EEG, but it will continue to be used for its advantages: ease of use and cost-effectiveness; freedom from problems associated with patient movement; and good temporal resolution in real time.

However, none of the newer imaging technologies is fully mature, and rapid change is still occurring. Technical developments in fMRI, MEG and event-related optical signals (EROS) may enable these to be used at deeper levels of the brain. Methods of interpreting imaging data, such as waveform and mathematical analyses, are improving constantly. Novel experimental design may overcome some technical limits and complementary, multi-modal studies offer possibilities yet to be fully explored. TMS studies can be carried out in an MRI machine, but MEG and MRI are difficult to use in conjunction with certain stimuli. Efforts to overcome this are under way but need time, effort and funding.
At present, there seems no prospect of getting imaging resolution down to the singlecell level, although the most powerful MEG and FMRI machines already operate at a resolution of a few millimetres. Single-unit recording in animals has always been the building block in neurology, but there are arguments that this may not continue to be an essential paradigm (see above).

By using PET, a functionally distinct area of the brain can be identified by high tracer uptake contrasting with low uptake in the surrounding tissue. With sufficient radioactive counts, the area can be visualised, however small it is in actual volume; thus, a very high resolution could be achieved with PET. For safety reasons, the number of repeat scans per individual is limited, and having to average across subjects reduces accuracy, because of individual differences in local cortical architecture. Nevertheless, within strict radiation exposure limits, repeat PET scans can be taken permitting, for example, changes in receptor binding to be measured (34).

There is great potential for PET as a neuropharmacological research tool. Radiolabelled ligands are available for opioid, dopamine, 5-hydroxytryptamine and nicotinic cholinergic receptors, but the repertoire needs to be developed further. Investment in this is required, and it can be time-consuming (4-5 years per ligand). However, once a large library of ligands is available, specific pharmacological questions about the human brain can be answered. Although the very fine anatomical detail which can be obtained from animal studies is not available, even at the level of resolution currently attained by imaging, useful questions can be answered. For example, in neuropathic and arthritic pain, it has been established by using PET with volunteers that the endogenous opiate system is switched on by chronic pain.

MRI is increasingly valuable for observing brain tissue in health and disease, and good progress is being made in establishing its specificity. More confidence in the results will come with time and familiarity, as well as with method optimisation (35) and with more data which relate images from living patients to pathology in post-mortem brain tissue. There has been a shortage of fresh post-mortem and surgical human brain tissue for this purpose, but some patients have given their advance consent for their brain 
tissue to be used after death, for improving MRI specificity (Figure 3).

A new technique has emerged in which cortically blind patients have had small electrode mounts implanted into their visual cortex. After a month or so, dendrites made contacts with the electrodes and visual sensations resulted. With further development, this approach is likely to yield new insights into the micro-architecture of the human brain.

\section{Ethical and safety limitations}

With human studies, ethical and safety barriers arise which are not considered by many scientists to be problematic in animal experiments. With some human research, it can be difficult to adopt a very rigorous scientific approach, because of limitations on what interventions are ethically acceptable.

There is potential to study disease as it progresses in patients, but knowledge of how

Figure 3: Magnetic resonance imaging scan of normal brain

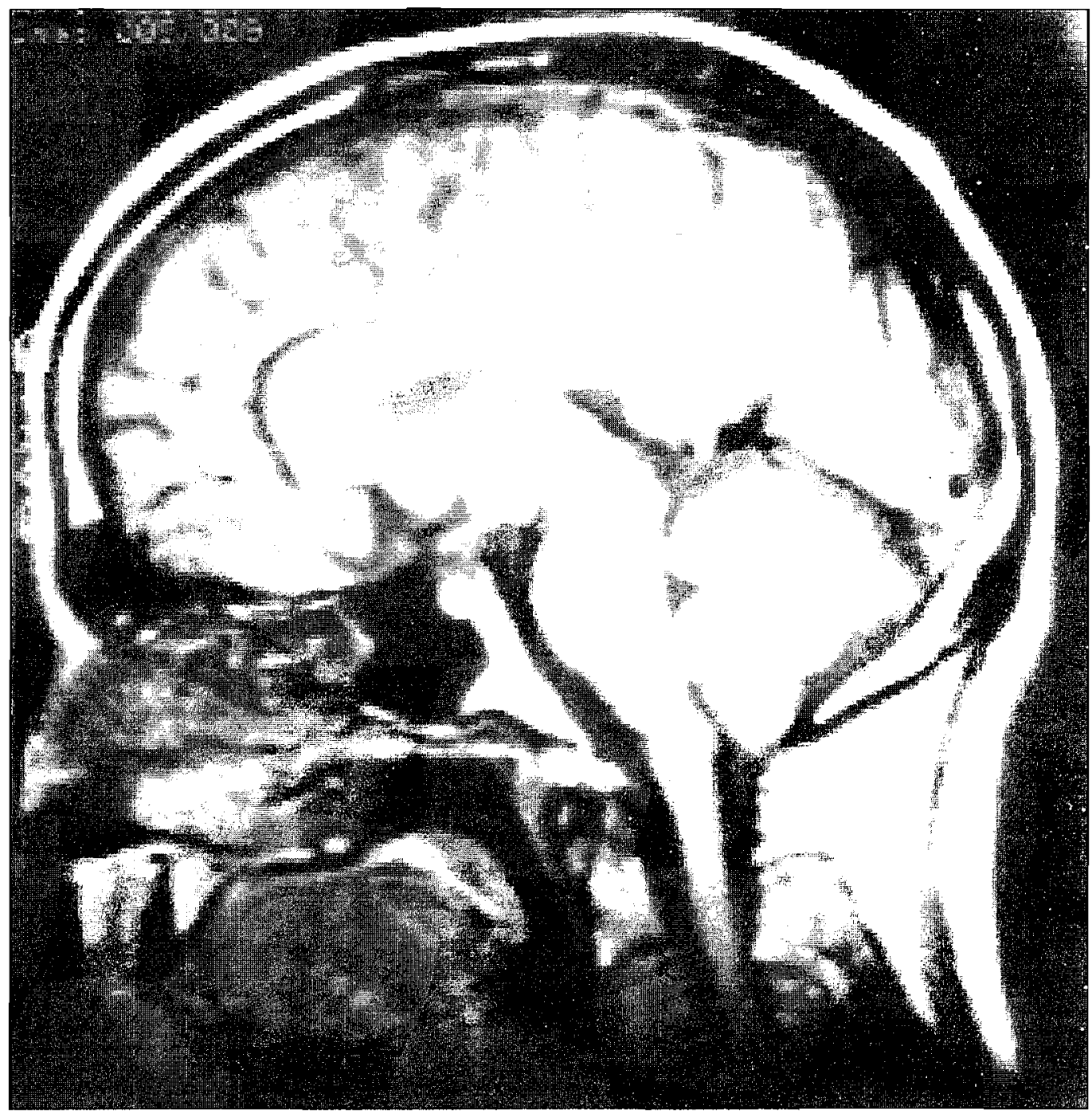


the individual patient's brain was functioning before illness is not usually available. To improve understanding, brain processes sometimes need to be modified or altered experimentally, and this cannot always be done ethically with human studies. Studies that aim to establish the limits of risk, such as toxicity testing or where results are difficult to predict, are likely to continue to be conducted in animals.

Although MEG and EEG are entirely noninvasive, the long-term risks, if any, of PET, MRI and TMS are not yet established. It is, of course, essential that volunteers should have a clear perception of risk as it is currently understood. With PET scans, the regulations restrict radiation exposure on an annual basis, and therefore limit repeat studies in healthy volunteers. When patients are scanned for diagnosis or therapy, higher radiation levels are permitted. Improvements in radiolabelling to reduce radiation exposure seem unlikely, but more-sensitive imaging equipment would help reduce exposure. With regard to the cumulative effects of PET scans, more information on the wholebody distribution of PET tracers in humans is needed.

\section{Limitations imposed by the research culture}

Imaging is relatively new and research culture can be slow to change. The perceived success of brain research with animals may create a delay in switching to humau studies. Hypotheses have traditionally been generated by using animal data, tending in turn to generate more animal experiments. Newer hypothesis-generating developments, such as in vitro work, computer simulations and human studies, may break this loop.

Sometimes, imaging results in humans generate new hypotheses that are still tested in animals, simply because animals have always been used to validate findings in humans. It is hard to justify this practice and, at least in some areas of brain research, animal models are becoming progressively less appropriate as technological advances enable human studies to be performed.

The attitudes of scientists to animal and human studies tend to vary in different disciplines and in different countries. In the USA, Japan and Germany, primate research continues to dominate, and in countries such as France the use of human volunteers is very restricted. In Britain, there has been a paradigm shift toward acceptance of the functional architecture model of the brain. Imaging results are generally held to be valid, and this may be linked to a move away from invasive animal work, which is especially prominent among younger scientists.

In the world of drug development, pharmaceutical companies can be conservative and bound by regulatory requirements: this is a barrier to change that has been observed in other areas of regulatory testing.

Although specialist meetings and journals are useful for method development, to maximise its potential, imaging should also be kept in the mainstream and should not be isolated as a separate field. In the past, the potential of methods to answer important questions was not always quickly recognised. Visual evoked potentials were "ghettoised" in EEG journals and EEG societies for a long time, and the community of researchers employing evoked potentials was relatively closed. Psychologists were largely unaware of the value of evoked potentials until they were "re-invented" as an imaging technique. Imaging should continue to address experimental questions which matter to other researchers and, indeed, it is now central to some fields, such as cognitive neuroscience.

\section{Funding limitations}

There are also barriers represented by the cost of some imaging facilities and the fund ing needed to develop them. Capital costs can be high, especially with PET, MEG and MRI. The availability of equipment can be limited, but once the capital investment has been made, each imaging experiment can be relatively inexpensive to perform.

Research institutes and funding bodies should be aware of the full cost of animal experiments, involving not just the purchase of animals (one monkey costs about $£ 3000$ ), but also the cost and/or space demands of animal housing, caging, feed, barrier facilities, animal staff, security and all the infrastructure and paperwork required to support animal research.

Funding to develop systems for new approaches can be difficult to obtain: the pressure is to do research with existing techniques. More funding for multidisciplinary collaborative groups could help, as would centres for developing techniques and training clinical researchers. It appears to some scientists that funding bodies tend to over- 
emphasise the limitations of human studies, and that research councils are dominated by basic scientists who regard animal work as the norm.

There are few pathways for biologists to develop clinical careers and little opportunity to obtain clinical training. The planned career path for academic clinical scientists is considered inadequate, and financial incentives are poor. Technology and methodology support staff (such as physicists, computer experts and mathematicians) are essential but, at present, universities make inadequate investment in these areas, even though the continued development of imaging depends on them, and current gains have been made on the basis of physics research done in the 1970s.

Funding organisations need to recognise the importance of key technical support staff, whose skills and expertise are in short supply. Funding bodies tend to expect these staff to be financed by the universities, but their posts are often the first to be cut, especially as their output is difficult to monitor. Without long-term funding for a minimum of five years and a reasonable career structure, highly qualified support staff are difficult to attract into academic research. This is a particular problem in imaging, where methods of data analysis and computing are relatively new and complex, and where interdisclipinary research is the norm. Moreover, team members need to be able to collaborate. In some institutes, physicists working on imaging techniques are isolated in the physics department, possibly unaware of the details of the questions the techniques are required to answer.

\section{Conclusions and Recommendations}

1. With animal experiments, it is essential to ensure that a critical appraisal is made at every stage of project development, especially to ask the following questions: "What is the ultimate purpose of the study?" and "Is it appropriate to address some or all of the key issues in healthy or patient volunteers, or by other non-animal means?" Researchers, funding bodies, Home Office inspectors and ethical review processes all have a responsibility to participate in this ongoing appraisal.
2. A flow diagram for this decision-making process should be developed for a range of research fields. This would be especially useful for lay members of ethical review processes, but would also help all participants to clarify their decision making.

3. Certain classes of research, such as cognitive neuroscience, are particularly amenable to the replacement of animals by human studies. In cognitive psychology, for example, much brain surface lesioning research and many regional activation experiments with electrode mats in animals could be replaced by human studies with TMS. Human studies employing a range of imaging methods are feasible and appropriate for studying psychology, at both the systems level and the global level, and could provide much of the data necessary in this field.

4. Some animal studies of sensory and motor function, brain plasticity and repair, and CNS pathologies can be replaced by integrated human imaging and in vitro research with human tissue. None of the newer technologies is fully developed, and rapid change is still occurring. Technical developments in fMRI, MEG and eventrelated optical signals (EROS) could permit these methods to be used at deeper levels of the brain.

5. Imaging permits human studies of disease evolution and treatment responses, particularly with brain tumours, multiple sclerosis, stroke and dementia. These studies are revealing the limitations of some traditional animal models. However, ethical concerns constrain the experimental interventions that can be conducted with human subjects.

6. Early CNS drug efficacy and metabolism studies in humans offer several benefits in relevance, time, cost and animal lives. Greater investment by the pharmaceutical industry in the development of mapping and imaging methods in humans would be very worthwhile.

7. The repertoire of radiolabelled ligands for PET research into specific receptors needs to be developed further. This can be time-consuming and requires investment. However, once a large library of ligands is available, very specific phar- 
macological questions about the human brain could be answerabled.

8. Human data are of ultimate relevance in medical and clinical fields. In human imaging studies, there is no species barrier problem; this is especially important in functional anatomy and drug effects. Imaging research on the human brain has provided new global information about integrated networks and has identified areas of unexpected relevance.

9. Imaging techniques are relatively novel, and the research culture tends to be slow to change. The perceived success of brain research with animals may create a delay in switching to human studies. Sometirnes, imaging data from humans generate new hypotheses that are still tested in animals. It is hard to justify this practice scientifically. In some areas of brain research, animal models are becoming progressively less appropriate as technological advances enable human studies to be performed.

10. Imaging modalities have different strengths and weaknesses with regard to temporal and spatial resolutions. Existing imaging methods are unlikely to reach the single-cell level of resolution, but this kind of knowledge may not be necessary. Better spatial resolution can be achieved in isolated tissue than in the whole brain. Various biochemical and pharmacological methods applied to human brain tissue ex vivo can complement imaging of the living brain, thus permitting the replacement of more animal experiments.

11. There is a pressing need for the wider availability of fresh human post-surgical and post-mortem brain tissue, for use in biochemical and pathological studies to validate aspects of MRI.

12. The integration of different imaging modalities can expand their potential and offer previously unavailable research possibilities. Integrated imaging studies with humans should be considered as a potential replacement of animal experiments when asking questions about the brain.

13. Imaging and related methods should not be "ghettoised", but should remain mainstream and should be applied to the scientific questions of interest across a range of disciplines. Meetings should be science-based and problem-based, as well as technique-based.

14. Although the initial capital investment for imaging facilities can be large, once they are established, subsequent human studies can be very productive and can generate data more swiftly than, and often as economically as, animal experiments. However, at the moment, capital costs are high for some equipment, the availability of which can be a limiting factor.

15. Highly qualified technological and methodological support staff are essential in imaging studies, which can span several disciplines. Methods of interpreting imaging data, such as waveform and mathematical analyses, are being improved, but expert staff are in short supply, and it is difficult to secure longterm funding for these types of posts. Appropriate funding and a career structure would help these staff to be retained and would ensure the continued development of imaging techniques.

16. Multidisciplinary research teams are required for maximum progress with imaging. Improvements in career pathways for biologists who want to do clinical studies and for clinicians to enter research would facilitate human studies.

17. Earmarked funding is needed for the development of imaging methodology, such as how to deliver stimuli in imaging environments. Funding bodies generally undervalue these crucial developmental aspects.

\section{Acknowledgements}

The workshop was supported by a grant from the Dr Hadwen Trust for Humane Research.

\section{References}

1. HMSO (1986). Animals (Scientific Procedures) Act 1986. London, UK: HMSO.

2. EEC (1986). Council Directive 86/609/EEC of 24 November 1986 on the approximation of laws, regulations and administrative provisions of the 
Member States regarding the protection of animals used for experimental and other purposes. Official Journal of the European Communities L358, 1-29.

3. Close, B., Combes, R., Hubbard, A. \& Illingworth, J., eds (1997). Volunteers in Research and Testing, 198pp. London, UK: Taylor \& Francis.

4. Home Office (1999). Statistics of Scientific Procedures on Living Animals: Great Britain 1998, $108 \mathrm{pp}$. London, UK: The Stationery Office.

5. Wilson, F.A. \& Rolls, E.T. (1993). The effects of stimulus novelty and familiarity on neuronal activity in the amygdala of monkeys performing recognition memory tasks. Experimental Brain Research 93, 367-382.

6. Hackett, T.A., Stepniewska, I. \& Kaas, J.H. (1999). Prefontal connections of the parabelt auditory cortex in macaque monkeys. Brain Research 817, 45-58.

7. Nakagawa, S., Mizuma, M. \& Kuchiiwa, S. (1998) The retinal projections to the ventral and dorsal divisions of the medial terminal nucleus and mesencephalic reticular formation in the Japanese monkey (Macaca fuscata): a reinvestigation with cholera toxin $\mathrm{B}$ subunit as an anterograde tracer. Brain Research 809, 198-203.

8. Gerhardt, G.A., Cass, W.A., Huettl, P., Brock, S., Zhang, Z. \& Gash, D.M. (i999). GDNF improves dopamine function in the substantia nigra but not the putamen of unilateral MPTP-lesioned rhesus monkeys. Brain Research 817, 163-171.

9. Zahrt, J., Taylor, J.R., Mathew, R.G. \& Arnsten, A.F.T. (1997). Supranormal stimulation of D1 dopamine receptors in the rodent prefrontal cortex impairs spatial working memory performance. Journal of Neuroscience 17, 8528-8535.

10. Bergson, C., Mrzijak, L., Smiley, J.F., Pappy, M. Levenson, $R$ \& Goldman-Rakic PS (1995) Regional, cellular, and subcellular variations in the distribution of D1 and D5 dopamine receptors in primate brain. Journal of Neuroscience $\mathbf{1 5}$ $7821-7836$

11. Miller, D.H. \& Frank, J.A. (1998). Magnetic resonance imaging techniques to monitor short term evolution of multiple sclerosis and to use in preliminary trials. Journal of Neurology Neurosurgery and Psychiatry 64, Suppl. 44-46.

12. Courtney, S.M., Petit, L., Maisog, J.M., Ungerleider, L.G. \& Haxby, J.V. (1998). An area specialized for spatial working memory in human frontal cortex. Science. New York 279, 1347-1351

13. Walsh, V. \& Cowey, A (1998). Magnetic stimulation studies of visual cognition. Trends in Cognitive Sciences 2, 103-109

14. Koepp, M.J., Gunn, R.N., Lawrence, A.D., Cunningham, V.J., Dagher, A., Jones, T., Brooks, D J Bench, C.J \& Grasby P. (1998) Evidence for striatal dopamine release during a video game. Nature 393, 266-268

15. Conturo, T.E., Lori, N.F., Cull, T.S., Akbudak, E., Snyder, A.Z., Shimony, J.S., McKinstry, R.C. Burton, H \& Raichle, M E (1999). Tracking neuronal fiber pathways in the living human brain. Proceedings of the Natzonal Academy of Sciences USA 96, 10,422-10,427

16. Anderson, S.J., Holliday, I.E., Singh, K.D. \& Harding, G.F. (1996). Localization and functional analysis of human cortical area V5 using magneto-encephalography. Proceedings of the Royal Society London - B. Biologucal Sciences 263.
$423-431$.

17. Hamdy, S., Aziz, Q, Rothwell, J.C., Hobson, A. \& Thompson, D.G. (1998). Sensorimotor modula tion of human cortical swallowing pathways Journal of Physiology (London) 506, 857-866.

18. Paus, T., Jech, R., Thompson, C.J., Comeau, R., Peters, T \& Evans, A.C. (1997). Transcranial magnetic stimulation during positron emission tomography: a new method for studyine connectivity of the human cerebral cortex. Journal of Neuroscience 17, 3178-3184

19. Werhahn, K.J., Kunesch, E., Noachtar, S. Benecke, R. \& Classen, J. (1999). Differential effects on motorcortical inhibition induced by blockade of GABA uptake in humans. Journal of Physiology (Loncton) 517, 591-597.

20. Fried, I., MacDonald, K.A. \& Wilson, C.L. (1997) Single neuron activity in human hippocampus and amygdala during recognition of faces and objects. Neuron 18,753-765

21. Clarke, S. (1994). Modular organization of human extrastriate visual cortex: evidence from cytochrome oxidase pattern in normal and macular degeneration cases. European Journal of Neuroscience $6,725-736$.

22. Dai, J., Swaab, D.F. \& Buijs, R.M. (1998). Recov ery of axonal transport in "dead neurons". The Lancet 351, 499-500.

23. Motluk, A. (1997). Twinning techniques to map the mind. New Scientist 2081, 26

24. Hamdy, S., Rothwell, J.C., Aziz, Q., Singh, K.D. \& Thompson, D.G. (1998). Long-term reorganisation of human motor cortex driven by short-térm sensory stimulation. Nature Neuroscience 1, $64-68$.

25. DeKosky, S.T. \& Scheff, S.W. (1990). Synapse loss in frontal cortex biopsies in Alzheimer's disease. correlation with cognitive severity. Arunals of Neurology 27, 457-464.

26. Laasonen-Balk, T., Kuikka, J., Viinamaki, H., Husso-Saastamoinen, M., Lehtonen, J. \& Tiihonen, J. (1999). Striatal dopamine transporter density in major depression. Psychopharmacology (Berlin) 144, 282-285

27. Baumgartner, C., Olbrich, A., Lindinger, G., Pataraia, E. Groppel, G., Bacher, J., Aull, S., Serles, W., Hoffman, M., Leutmezer, F., Czech, T., Prayer, D., Pietrzyk U. Asenbaum, S. \& Podreka, I. (1999). Regional cerebral blood flow during temporal lobe seizures associated with ictal vomiting: an ictal SPECT study in two patients. Epilepsia 40, 1085-1091.

28. Aquilonius, S.M. (1991). What has PET told us about Parkinson's disease? Acta Neurologica Scandinavica, Supplemenl 136, 37-.39

29. Farde, L. (1996). The advantage of using position emission tomography in drug research. Trends in Neurosciences 19,211-214.

30. Grasby, P. (1998). Psychiatric iliness: a PET subject. $M R C$ News 77, 28-32

31. Chipkin, R.E., Berger, J.G., Billard, W., lorio, L.C., Chapman, R. \& Bannett, A (1988) Pharmacology of SCH 34826, an orally active enkephalinase inhibitor analgesic. Journal of Pharmacology and Experimental Therapeutucs 245, 829-838.

32. Christensen, J.D Yurgelun-Todd, D.A., Babb, S.M., Gruber, S.A., Cohen, B.M. \& Renshaw, P.F. (1999). Measurement of human brain dexfinflu ramine concentration by $19 \mathrm{~F}$ magnetic resonance 
netic resonance spectroscopy. Brain Research $834,1-5$.

33. Jones, A.K. (1999). The contribution of functional imaging techniques to our understanding of rheumatic pain. Rheumatic Diseases Clinic of North America 25, 123-152.

34. Jones, A.K.P. Cunningham V.J. Ha-Kawa, S Fujiwara, T., Luthra, S.K. \& Jones, T. (1994).
Changes in central opioid receptor binding in relation to inflammation and pain in patients with rheumatoid arthritis. British Journal of Rheumatology 33, 909-916.

35. Tofts, P.S. (1998). Standardisation and optimisation of magnetic resonance techniques for multicentre studies. Journal of Neurology Neurosurgery and Psychiatry 64, Suppl. 37-43. 